SUBJECT AREAS:

BIOCHEMICAL REACTION NETWORKS

PROTEOMICS

METABOLIC DISORDERS

COMPUTATIONAL MODELS

Received

24 September 2013

Accepted

13 January 2014

Published

29 January 2014

Correspondence and requests for materials should be addressed to

N.J. (neema@ucsd. edu)

\section{Network reconstruction of platelet metabolism identifies metabolic signature for aspirin resistance}

\author{
Alex Thomas ${ }^{1,4}$, Sorena Rahmaniann ${ }^{2,4}$, Aarash Bordbar ${ }^{2,4}$, Bernhard Ø. Palsson ${ }^{2,3,4}$ \& Neema Jamshidi ${ }^{3,4,5}$
} ${ }^{1}$ Department of Bioinformatics and Systems Biology, ${ }^{2}$ Department of Bioengineering, ${ }^{3}$ Institute of Engineering and Medicine,
${ }^{4}$ University of California, San Diego, 9500 Gilman Drive, Mail Code 0412, La Jolla, CA 92093-0412, ${ }^{5}$ Department of Radiological
Sciences, University of California, Los Angeles, BOX 951721, Los Angeles, CA 90095-1721.

Recently there has not been a systematic, objective assessment of the metabolic capabilities of the human platelet. A manually curated, functionally tested, and validated biochemical reaction network of platelet metabolism, iAT-PLT-636, was reconstructed using 33 proteomic datasets and 354 literature references. The network contains enzymes mapping to 403 diseases and 231 FDA approved drugs, alluding to an expansive scope of biochemical transformations that may affect or be affected by disease processes in multiple organ systems. The effect of aspirin (ASA) resistance on platelet metabolism was evaluated using constraint-based modeling, which revealed a redirection of glycolytic, fatty acid, and nucleotide metabolism reaction fluxes in order to accommodate eicosanoid synthesis and reactive oxygen species stress. These results were confirmed with independent proteomic data. The construction and availability of iAT-PLT-636 should stimulate further data-driven, systems analysis of platelet metabolism towards the understanding of pathophysiological conditions including, but not strictly limited to, coagulopathies.

\footnotetext{
$\Lambda$

lthough it is one of the simplest and smallest human cells, the platelet has a rich biochemical repertoire and likely plays an underappreciated role in a range of human diseases. Herein we focus on the role platelet metabolism plays in the incompletely understood phenomenon of aspirin resistance. Aspirin (acetyl salicylic acid, ASA) is one of the most common medications taken today and is available over the counter, but is also prescribed at higher doses as a prophylactic, 'first line' anticoagulant, as it has been shown to reduce vascular mortality, myocardial infarction, and stroke among high-risk patients with atherothrombotic disease ${ }^{1}$. Aspirin inhibits cyclooxygenase-1 (COX-1) in platelets, inhibiting the potent vasoconstrictor and platelet agonist thromboxane $\mathrm{A}_{2}\left(\mathrm{TXA}_{2}\right)$. Although the pharmacological mechanism of aspirin has been identified and its health benefits are widely accepted, it has been estimated that $10-20 \%$ of aspirin-treated patients may experience diminished or absent response to aspirin treatment ${ }^{2}$. Various studies have explored the prevalence of aspirin resistance in patient populations $s^{3,4}$, but there have not been any cell-scale, quantitative, mechanistic delineations of the effect of diminished cyclooxygenase activity on platelet metabolism.

Systems biology catalogues knowledge in a framework enabling mechanistic insight into causes and effects of resulting biological phenotypes, allowing for the contextualization of datasets which subsequently promote the predictive capabilities of biological models. There has been demonstrated utility in using metabolic reconstructions as scaffolds for systems modeling of biological organisms $s^{5}$, with applications ranging from metabolic engineering ${ }^{6}$ to studying human disease ${ }^{7}$ and predicting responses to therapeutic drugs ${ }^{8-11}$. Although smaller models exist for platelets which define signaling cascades ${ }^{12}$ or fluid dynamics ${ }^{13}$, in order to systematically assess the characteristics of platelet metabolism we constructed iAT-PLT-636, a cell-scale, mass and charge balanced representation of platelet metabolism that is built and functionally verified from proteomic, metabolomic, fluxomic, and physiological data. iAT-PLT-636 provides an assessment of platelet metabolism that heretofore has not been described and further, may disclose a potential influence on a variety of acute and chronic disease conditions. The global view of metabolism afforded by iAT-PLT-636 provides an ideal scaffold for the analysis of high throughput datasets and understanding the systems-level context of the outcome of small-scale experiments. Thus, a biochemical, cell-scale signature for ASA-resistance was derived using iAT-PLT-636 with physiological constraints simulating ASA response, ultimately providing a mechanistic overview of the shifts in flux through reaction pathways for the ASA-resistant platelet.
} 


\section{Results}

Scope and content of iAT-PLT-636. iAT-PLT-636 was constructed in a systematic, quality controlled manner with quality control/ quality assurance (QC/QA) measures using 33 human platelet proteomic studies and 354 peer reviewed articles in the literature that were used to define the platelet biochemical network based on the global reconstruction of human metabolism (Figure 1 and Methods). As the first large-scale, mass balanced model of platelet metabolism, iAT-PLT-636 presents a breadth of platelet metabolism that has not been captured and perhaps not even recognized before in previous studies (Figure 2). Network content includes 1,008 reactions (biochemical transformations, intracellular transporters, and extracellular transporters), 739 compartment-specific metabolites, 225 proteins, and represents 636 genes contained in Recon $1^{14}$. Approximately one-third of the reactions have proteomic as well as bibliomic (primary literature) support. Proteomic support for extracellular and intracellular transport reactions is lacking, and thus primary literature sources provided the majority of the evidence for transport reactions. Conversely, proteomic studies provided further insight into the true scope of amino acid, carbohydrate, fatty acid (elongation, activation and oxidation), and nucleotide metabolism, providing potential missing links in the energy metabolism of the platelet ${ }^{15,16}$.

Gene-protein-reaction (GPR) relationships define the association between genes, metabolic enzymes, and the biochemical transformations that they ultimately facilitate ${ }^{17}$. These relationships also serve as a scaffold, enabling the integrated analysis of various high throughput data in iAT-PLT-636 $6^{18} .457$ reactions have a disease associated SNP, many of which have known therapeutic drug targets (Figure 3). 403 genetic variant diseases associated with 247 genes (Online

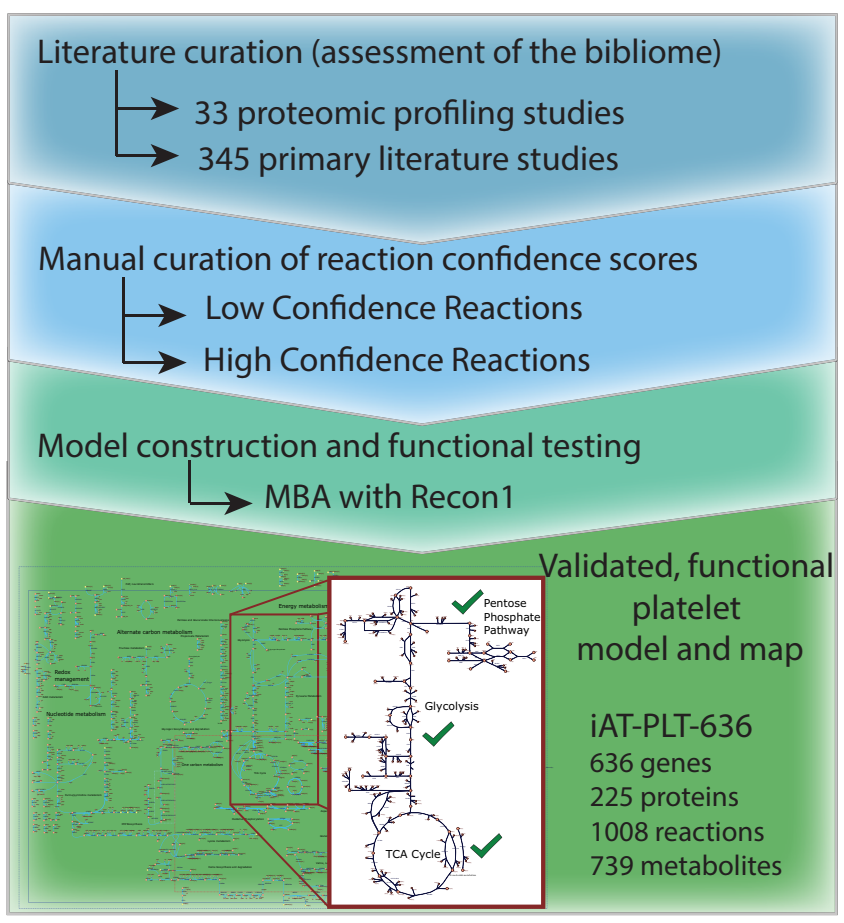

Figure 1 The platelet reconstruction was primarily built from proteomic and literature references on platelet metabolism. The references were used to assign high and low evidence to reactions that could be a part of platelet metabolism. This reaction specification and the human reconstruction were used in conjunction with a modified version of $\mathrm{MBA}^{47}$ to build a model for platelet metabolism. To actively curate and verify the reaction pathways reconstructed with $\mathrm{MBA}$, a metabolic reaction map was drawn and map-guided subsystem verification was done until a concise, high-evidence model for platelet metabolism was reconstructed.
Mendelian Inheritance in $\mathrm{Man}^{19}$ ) are encompassed within the purview of the model. Moreover, there are 231 FDA approved drugs that are associated with model enzymes, of which 183 overlap with the set of disease-associated SNP reactions (Figure 3). The ratio of drug targets to the total number of reactions is 0.23 for iAT-PLT-636 and 0.13 for the human reconstruction, indicating there is an increased density of druggable targets in the platelet model (Fisher's exact test, $p=3.3^{*} 10^{-13}$ ). An enrichment analysis for drug categories with targets in iAT-PLT-636 for FDA approved drugs in the DrugBank database ${ }^{20}$ reveals a set of categories that all have literature-validated roles in affecting platelet metabolism (Supplement) identifying 20 non-exclusive categories (Table 1).

Hierarchical characterization of iAT-PLT-636. The size and complexity of iAT-PLT-636 as assessed by the dimensions of the stoichiometric matrix and the size of the steady state flux solution space, places the platelet model in between two other human blood related cell metabolic networks, mature erythrocytes and macrophages (Supplemental Figure 1). The reaction dependencies within iAT-PLT-636 are too numerous and complex to fully appreciate simply from looking at a map (Supplemental Figures); objective, analytical and simulation based methods are needed to characterize the dependencies in the model. Functional relationships between reactions in metabolic networks can be calculated in numerous ways ${ }^{21}$; we employed established methods to identify perfectly correlated as well as partially correlated (or coupled) sets of reactions ${ }^{22,23}$ (see Methods, Figure 4A). Perfectly correlated reaction sets are groups of reactions in which the reactions are correlated (or anti-correlated) for a specified set of conditions. Coupled reactions account for the set of relationships in which a non-zero flux in one reaction implies a non-zero flux in another, although the range and magnitude of the fluxes may vary independently. Network content in iAT-PLT-636 was the assessed in terms of these relationships between different reactions. Reaction set connectivity between reactions was defined as the inclusion of a reaction in a coset (correlated reaction set) ${ }^{24}$ or coupled ${ }^{22,23}$ with other reactions (Figure 4A).

iAT-PLT-636 has 179 cosets and 460 coupled reaction sets (Supplement), with an average of 3.39 reactions and 83.57 reactions, respectively. While perfectly correlated cosets are mutually exclusive sets of reactions, coupled reactions are not. On average, 16.43 reactions were shared between coupled reaction sets and 38 reactions per coupled set (median of 28). Figure 4B illustrates the numerous complex dependencies of reactions in the model which largely center around transport reactions for substrates and products related to ATP generation. Clustering the set of cosets and flux coupled reaction sets resulted in three principle clusters of reaction subsystems (Figure 4C). Cosets are colored red and appear adjacent to the diagonal, whereas coupled reactions are colored green and appear in the farther off-diagonal entries. The first cluster of reactions is highly interconnected, with many connections to other reactions in the network. The second cluster contains key reaction systems in platelet hemostatic cascades (prostaglandin and serotonin synthesis) as well as specialized metabolism (neurotransmitter reuptake, glutathione synthesis, and mitochondrial exchange). Canonical pathways involve groups of reactions, some of which may be coupled, others of which may be in cosets, and still other reactions which are in neither. An example of this is the prostaglandin anabolic pathway (Figure 4D), which includes cosets, coupled reactions, as well as noncoupled reactions. Cluster three consists of phospholipid metabolism and is dominated by fatty acid metabolism, and the redundancies amongst these reactions make them uncoupled from most of the metabolic network, however many of these reactions are linked to ATP maintenance in the energy exchange group.

Identifying an ASA-resistant metabolic signature. iAT-PLT-636 was used to investigate the differences between platelet metabolism 


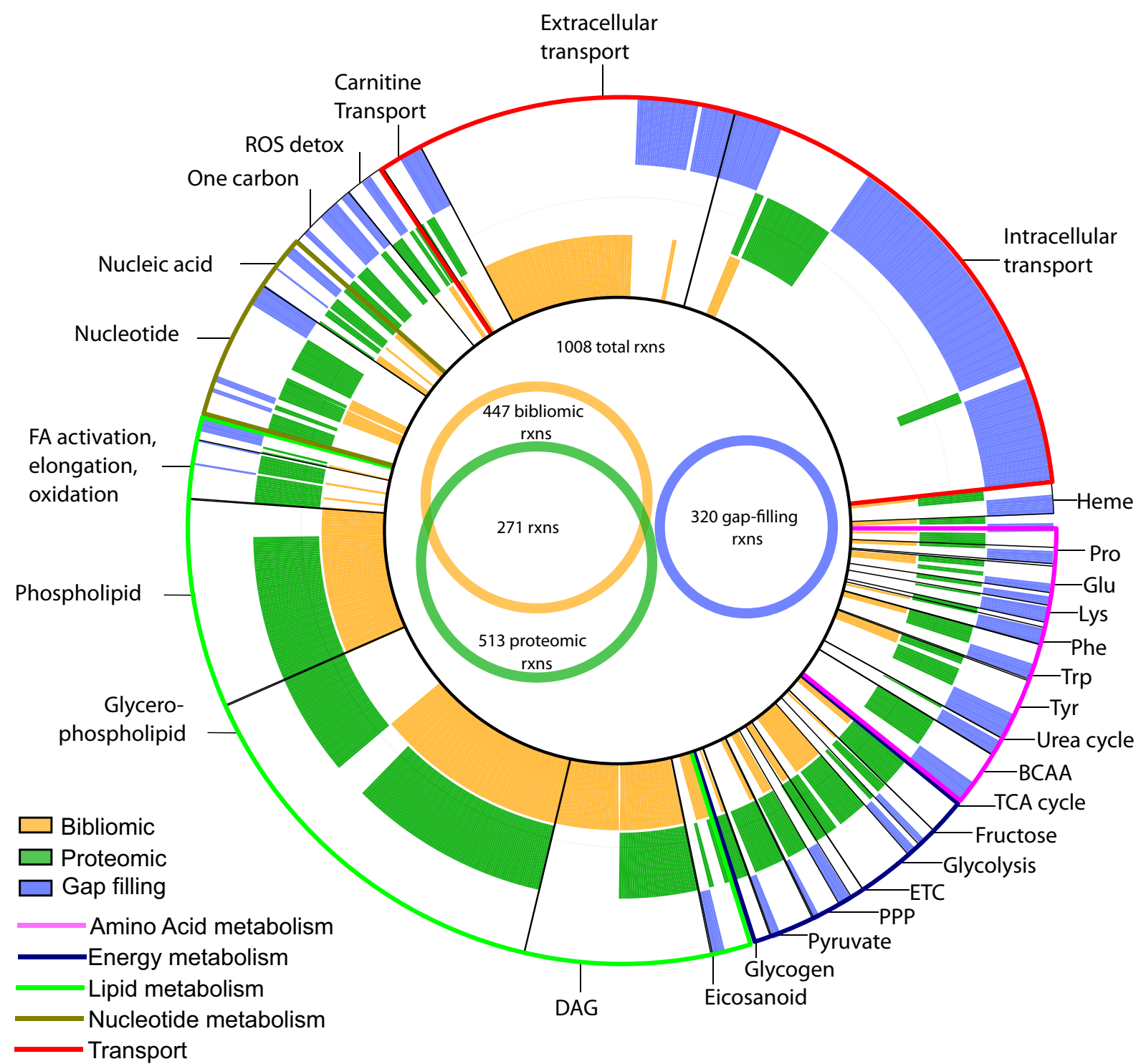

Figure 2 Content and evidence supporting the 1008 reactions in iAT-PLT-636. All reaction subsystems with over five reactions are highlighted to show the breadth of platelet metabolism; reaction subsystems are further grouped under five parent subsystems (amino acid metabolism, energy metabolism, lipid metabolism, nucleotide metabolism, transport). Abbreviations: Standard abbreviations are used in the figures such as common three letter amino acid abbreviations, BCAA = branched chain amino acid (i.e. valine, leucine, isoleucine metabolism), ETC = electron transport chain, PPP pentose phosphate pathway, DAG - diacylglycerol, FA - fatty acid, ROS - reactive oxygen species; TCA - tricarboxylic acid cycle.

in normal (ASA-sensitive, ASA-s) and ASA-resistant (ASA-r) individuals. Time course metabolomic and fluxomic ([14C]-labeled metabolites, see Methods) datasets generated from platelets in human plasma at $37^{\circ} \mathrm{C}^{15,25-28}$ were incorporated into iAT-PLT-636 as constraints on the metabolic reaction fluxes thereby constraining the feasible solution space of the metabolic network. Two different models were built for ASA-s and ASA-r individuals by varying the thromboxane $\mathrm{A}_{2}\left(\mathrm{TXA}_{2}\right)$ secretion flux, whose urinary byproduct is believed to be a clinically measurable indicator of ASA resistance ${ }^{3}$ (Methods). Reaction flux phenotypes were calculated via randomized sampling of the feasible flux states for the ASA-s and ASA-r platelet models ${ }^{29,30}$. Reaction fluxes that were significantly different (Wilcoxon rank-sum test, $p<0.05$ ) between the two conditions defined the ASA-r signature (Figure 5 and Methods). There is an observed diversion of NADPH and oxygen for prostaglandin synthesis which induces increased flux through the oxidative PPP while decreasing flux through aerobic ATP generation pathways in ASA-r platelets (Supplement).
In order to further validate and test the model predictions, we analyzed targeted proteomic data from ASA-s and ASA-r patient cohorts with stable coronary ischemic heart disease ${ }^{31}$, by determining if the reaction flux changes in the models could predict the measured proteomic differences in the clinical samples. During coronary ischemic insults, one consequence of decreased cardiac perfusion is an acute increase in oxidative stresses via elevated hydrogen peroxide levels ${ }^{32}$. Disease phenotypes can be evoked through biochemical flux demands $s^{33}$, so an increase in the hydrogen peroxide uptake was applied to the ASA-s and ASA-r models in order to simulate an increased ROS (reactive oxygen species) load. 150 metabolic reactions were significantly altered between ASA-s and ASA-r due the same ROS load (Figure 5 and Supplement).

Left null space pools of stoichiometric matrices represent groups of metabolites with conserved moieties in the network (i.e., metabolites or functional groups that are neither synthesized nor degraded), so the sum totals of the metabolites do not change ${ }^{34-36}$. Thus the left null space can be used to decipher seemingly unrelated changes in 
A.

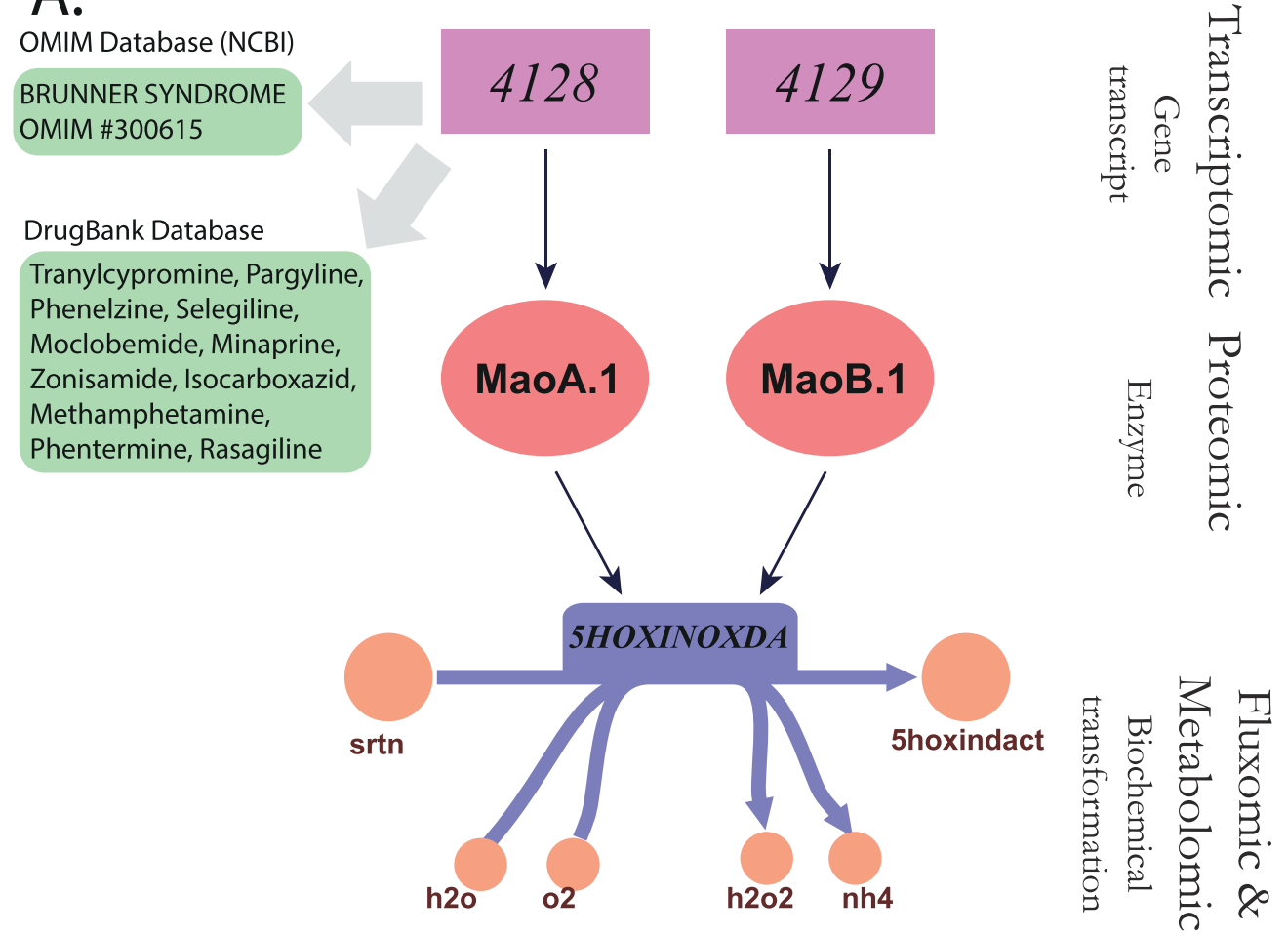

B.

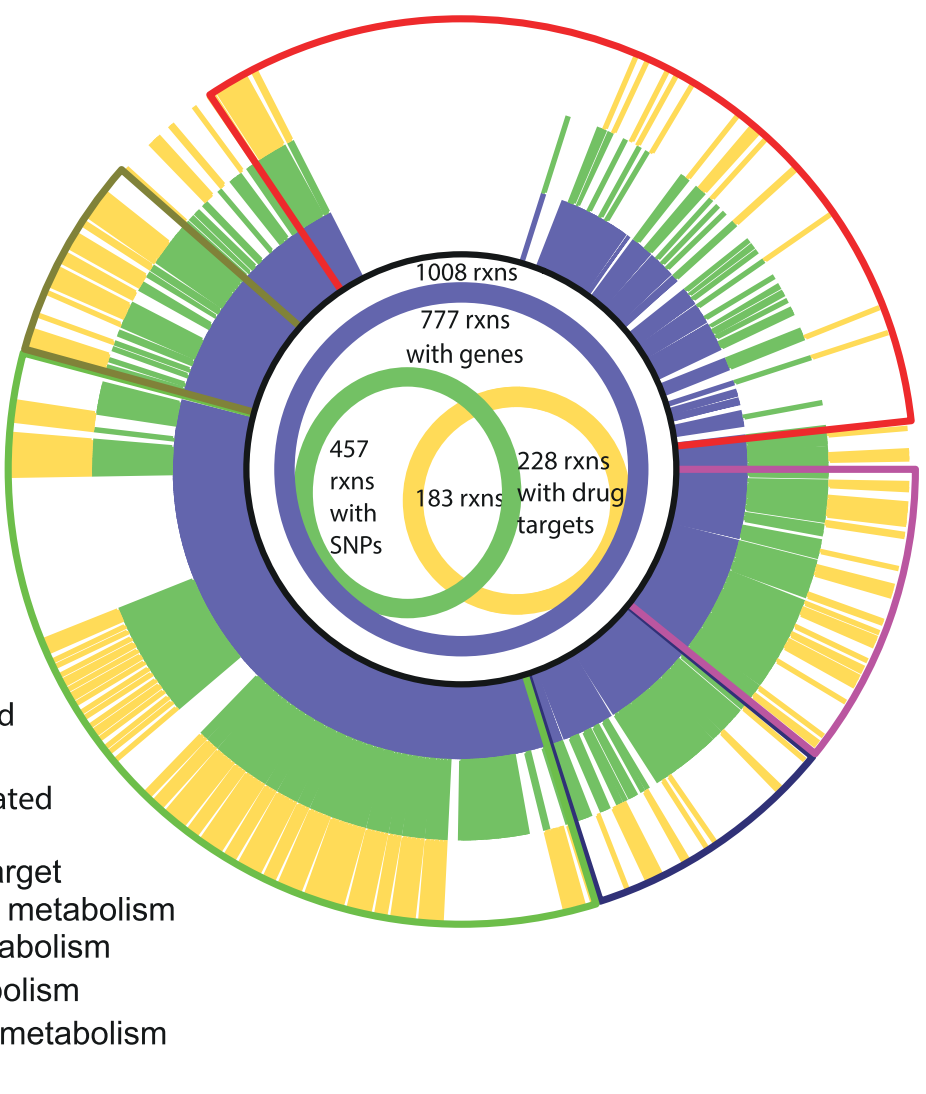

Figure 3 Platelet model properties. GPR (Gene-protein-reaction) relations from gene to reaction via drug targets and SNPs are shown to represent the information density of iAT-PLT-636 and provide the motivation for conducting mechanistic omics-mapping studies on platelet metabolism. (A) Example of a simple GPR for monoamine oxidase (5-hydroxytryptamine:oxygen oxidoreductase) which metabolizes serotonin, demonstrating the hierarchical levels of biological data integration in iAT-PLT-636. The thin black arrows represent the hierarchical structure between genes, proteins, enzymes, and metabolites. The block arrows highlight the identifiers used to query the different databases. (B) The innermost ring (blue) denotes whether the reaction has an explicit association with a known gene. The second (green) and third (yellow) rings highlight whether the associated gene with the reaction has a disease-associated SNP, $\mathrm{OMIM}^{19}$, or known drug target, DrugBank ${ }^{20}$, respectively. 
Table 1 | Enriched non-mutually exclusive Drug Categories (Fisherexact test with Bonferroni-Hochberg correction, $\alpha=0.05$ ) in the platelet model with respect to all other approved drugs in DrugBank

\begin{tabular}{lc} 
Drug Category & Corrected p-value \\
\hline Cyclooxygenase Inhibitors & $2.76 \mathrm{E}-18$ \\
Phosphodiesterase Inhibitors & $5.15 \mathrm{E}-11$ \\
Adrenergic Uptake Inhibitors & $6.48 \mathrm{E}-11$ \\
Vasodilator Agents & $7.92 \mathrm{E}-10$ \\
Nonsteroidal Anti-inflammatory Agents (NSAIAs) & $1.24 \mathrm{E}-09$ \\
Serotonin Uptake Inhibitors & $7.18 \mathrm{E}-07$ \\
Antidepressants & $4.04 \mathrm{E}-06$ \\
Antidepressive Agents & $6.62 \mathrm{E}-05$ \\
Cardiotonic Agents & $1.96 \mathrm{E}-04$ \\
Antidepressive Agents, Second-Generation & $6.04 \mathrm{E}-04$ \\
Thiadiazines & $1.24 \mathrm{E}-03$ \\
Dopamine Uptake Inhibitors & $1.24 \mathrm{E}-03$ \\
Diuretics & $1.26 \mathrm{E}-03$ \\
Antidepressive Agents, Tricyclic & $5.41 \mathrm{E}-03$ \\
Antipyretics & $7.68 \mathrm{E}-03$ \\
Monoamine Oxidase Inhibitors & $7.68 \mathrm{E}-03$ \\
Diuretics, Thiazide & $7.70 \mathrm{E}-03$ \\
Carbonic Anhydrase Inhibitors & $7.70 \mathrm{E}-03$ \\
Anesthetics, Inhalation & $7.70 \mathrm{E}-03$ \\
Norepinephrine-Reuptake Inhibitors & $7.70 \mathrm{E}-03$ \\
\hline
\end{tabular}

fluxes within the network. We analyzed the left null space metabolite pools to characterize the differences between the ASA-s and ASA-r flux states (Methods, Supplement, and Figure 5). The connection between $\mathrm{TXA}_{2}$ inhibition and the altered redox state is through the link between the NADP moiety in the cytoplasm and the endoplasmic reticulum (which is independent of the mitochondrial NADPH/ NADP pool). Changes in the NADH/NAD pools in the cytoplasm and mitochondria move in opposite directions, a result of the increased diversion of flux for prostaglandin synthesis in the ASA$\mathrm{r}$ model. Shifts in the CoA, Ubiquinone 10, and glutathione pools (i.e. changes in the 'charge ratios'), indicate flux diversions from fatty acid metabolism, oxidative phosphorylation, and redox management, respectively. In addition to the increased prostaglandin synthesis, ASA-r exhibits increased flux through glycolysis and nucleotide salvage pathways with corresponding decreases in redox management compared to ASA-s (Figure 5).

Patient data presented by Mateos-Cáceres et al showed an increase in glyceraldehyde 3-phosphate dehydrogenase (GAPD), a decrease in 1,6-bisphosphate aldolase (FBA), a decrease in glutathione Stransferase (GST), and a non-significant decrease in triose phosphate isomerase (TPI) for ASA-resistant platelets. These results were consistent with ASA-r model predictions, which exhibited increased fluxes through GAPD and TPI (Wilcoxon rank-sum test, $p<$ 0.05); the flux through FBA decreased, but was not significant. Simulation results predicting a significantly reduced flux through glutathione ROS metabolism and superoxide dismutase in ASA-r platelets and an inability of ASA-r platelets to sustain the same degree of hydrogen peroxide insults as ASA-sensitive platelets were confirmed with reduced GST expression.

\section{Discussion}

In this manuscript we provide a systems characterization of platelet biochemistry through the accomplishment of four objectives; 1 ) construction of a data-driven, manually curated, high quality network reconstruction of platelet metabolism, iAT-PLT-636, 2) assessment of network content by mapping to SNP associated diseases and drug targets, 3) elucidation of the hierarchical functional dependencies within the network, and 4) analysis of the aspirin resistance phenomenon with identification of characteristic biochemical signatures.
Although the platelet has a central role in the blood coagulation cascade, the broad spanning biochemical and metabolic capabilities allude to a potentially profound influence on organismal physiology and possible role in acute as well as chronic diseases. This is highlighted by the broad range of SNP associated diseases that have enzymes within core platelet metabolism (Figure 2). Moreover there is a broad scope of drug categories that span medications used in cardiology, rheumatology, psychology, neurology, nephrology, infectious disease, and anesthesiology. These results suggest potential 'off-organ' drug effects, including side-effects as well as off-target drug effects that may be important when evaluating existing and/ or new medications $s^{9,10}$.

A frequent challenge in the analysis of biochemical networks is the characterization of reaction and metabolite dependencies under specified conditions; there exist numerous ways to express these dependencies at feasible flux states of the networks ${ }^{21,22,24,37}$. For the platelet we focus on identification of perfectly correlated reaction sets (which simplify the network by collapsing reactions into modules), but also consider their interactions with other reactions that are coupled to them ${ }^{22,23,38}$. iAT-PLT-636 contains 1008 reactions and transporters with a 289 dimension (right) null space, indicating of a richness of metabolic capabilities. The cosets assist in reducing this complexity by creating reaction modules highlighting where the degrees of freedom within the solution space of the network lie. The coupled set of reactions (Figure 4C off diagonal elements) then allows one to see which reactions affect one another. The hierarchical structure and dependencies within the network highlighted in Figure 4 (Panels C and D) illustrate that it is not a trivial task to determine an intervention by simply blocking a particular reaction. The interplay of reactions must be taken into account when trying to identify new potential therapeutic targets. The role of neurotransmitters on platelet metabolism has been largely unexplore ${ }^{39}$ and the second reaction cluster highlights some of the different areas which may affect neurotransmitter re-uptake in the platelet, including the metabolism and transport of tyrosine, ascorbate, urea cycle intermediates, and high energy phosphate metabolites.

As a specific case study, ASA resistance was analyzed from the expanded view of metabolism provided by iAT-PLT-636. Although platelet turnover has been suggested to be a mechanism for ASA resistance ${ }^{15,40-43}$, the observed altered flux states from the simulations, as well as measured changes in platelet fluxes ${ }^{3}$, suggest that ASA resistance is attributable to more than just increased turnover of otherwise normally functioning platelets. Fluxomic data generated under physiological conditions (human plasma at $37^{\circ} \mathrm{C}$ ) was incorporated as quantitative flux constraints in order to generate aspirin sensitive and resistant models, ASA-s and ASA-r, respectively ${ }^{15,25-28}$. With these models in hand, clinical data was analyzed from patients with coronary ischemic heart disease ${ }^{31}$.

The ASA-r model predicted increases in GAPD and TPI, decreased FBA, and reduced ROS disposition via glutathione related pathways (compared to the ASA-s model). These predictions confirmed through targeted proteomic measurements of GAPD, TPI, and GST by Mateos-Cáceres et al. Interestingly, Mateos-Cáceres et al did not have a clear rationale for the seemingly contradictory concentration changes of the glycolytic enzymes. However, with iATPLT-636, the resulting flux pattern was easily accounted for as a redirection of carbon flux through the PPP. These results also affirm Mateos-Cáceres et al's proposed mechanism for reduced protection against ROS species in ASA-r platelets compared to ASA-s platelets, although model simulation results show additional propagated effects with reduction in flux through superoxide dismutase reactions and an almost significant $(p=.054)$ decrease in flux through catalase. Mateos-Cáceres et al purified and measured 5 oxidative stress proteins, of which GST had a significant decreased protein expression. Predictions of the model simulations reached a similar conclusion, supporting the role of glutathione metabolism in redox 
A.

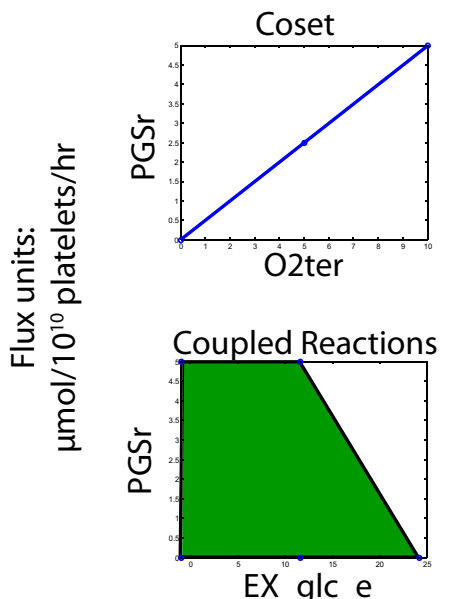

B.

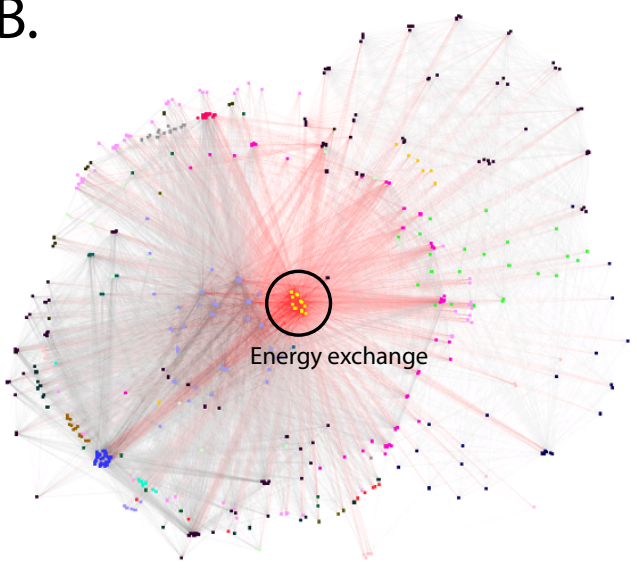

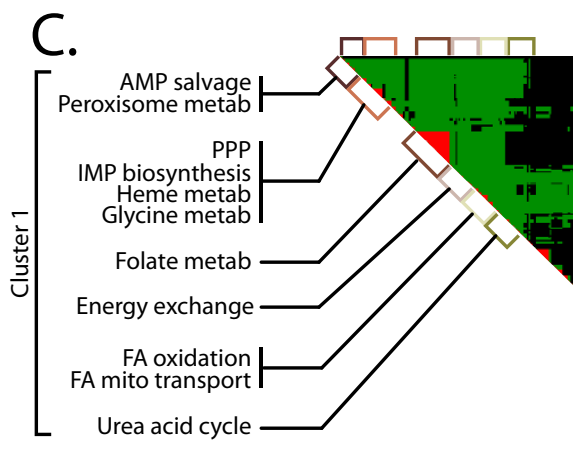

긴.

Prostaglandin synthesis
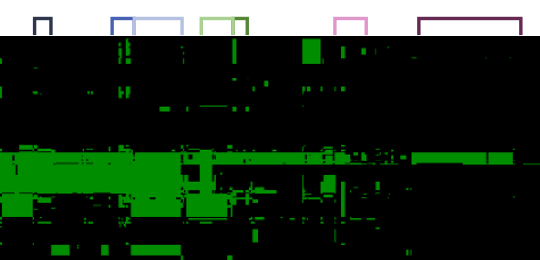

Mitochondrial $\mathrm{O}_{2}$, ATP exchange

Neurotransmitter Reuptake
Tyrosine, ascorbic metab

Glutamate metab
Glutathione metab

Glutathione metab

Serotonin metab

Pyruvate metabis
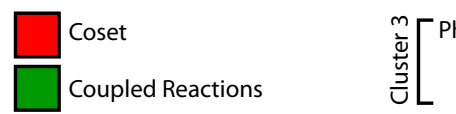

m Phospholipid degradation

Sphingosine metab

Phosphoinositol metab

D.

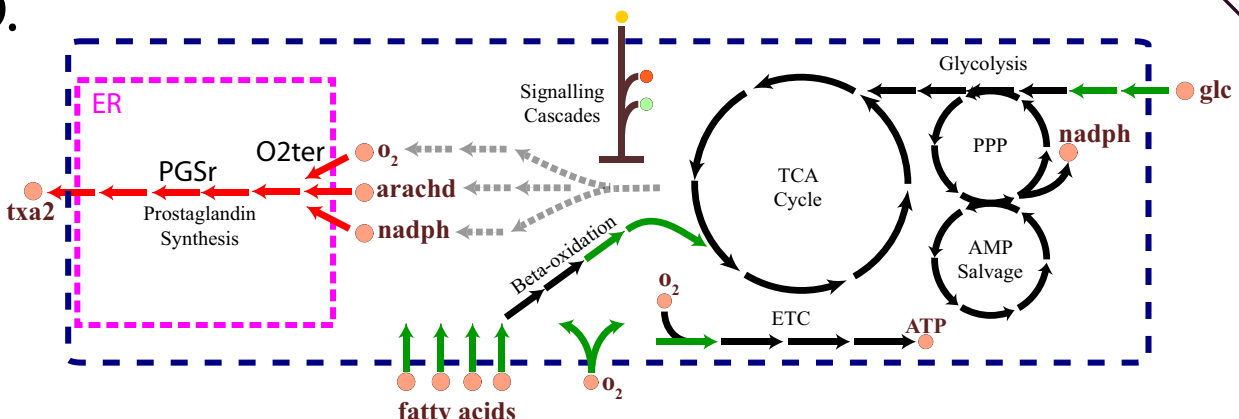

Figure 4 Cosets, flux coupling, and reaction connectivity in iAT-PLT-636. (A) Perfectly correlated reaction sets and coupled reaction sets were calculated for iAT-PLT-636. (B) Connectivity between the hard coupled and partially correlated reaction sets reveal energy metabolism related reactions and metabolites at the center of the coupling and correlations between reactions, in terms of viewing degree connectivity with a force-directed layout of reactions in the model (network map constructed with Cytoscape ${ }^{53}$ ). Various colors represent subsystems as defined in Figure 2. (C) Hierarchical clustering of the correlated reaction sets and coupled reaction sets reveal three tiers (clusters) of connectivity. Dominant metabolic subsystems within each cluster are highlighted. (D) Cartoon schematic of the prostaglandin synthesis pathway and related pathways with corresponding coset and coupled reactions. The final synthesis steps for prostaglandins are contained in a coset. Associated reactions contributing substrate precursors to the pathway involve reactions coupled to central metabolic pathways. Coset reactions are colored in red and coupled reactions are green. 
A.

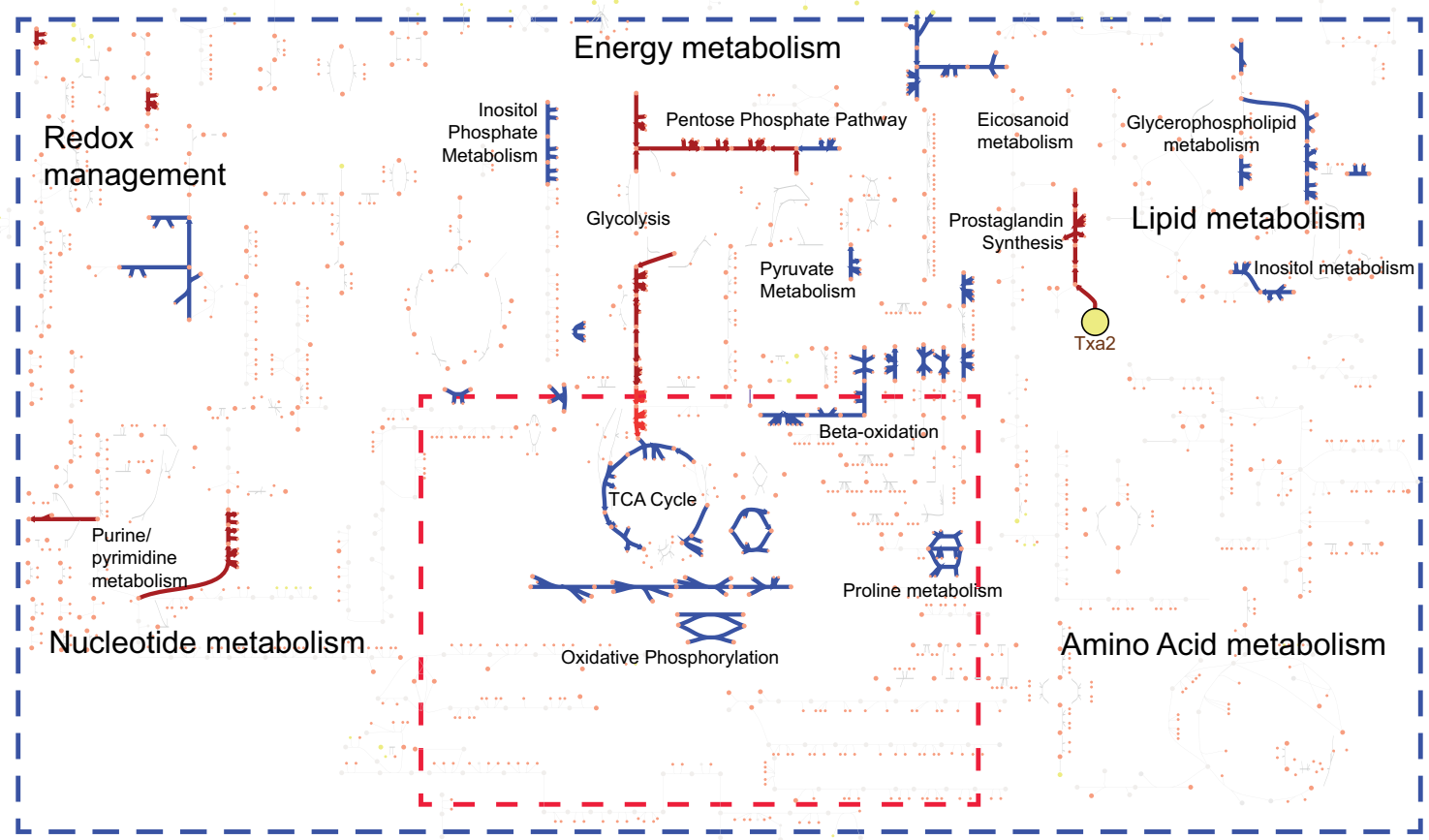

B.
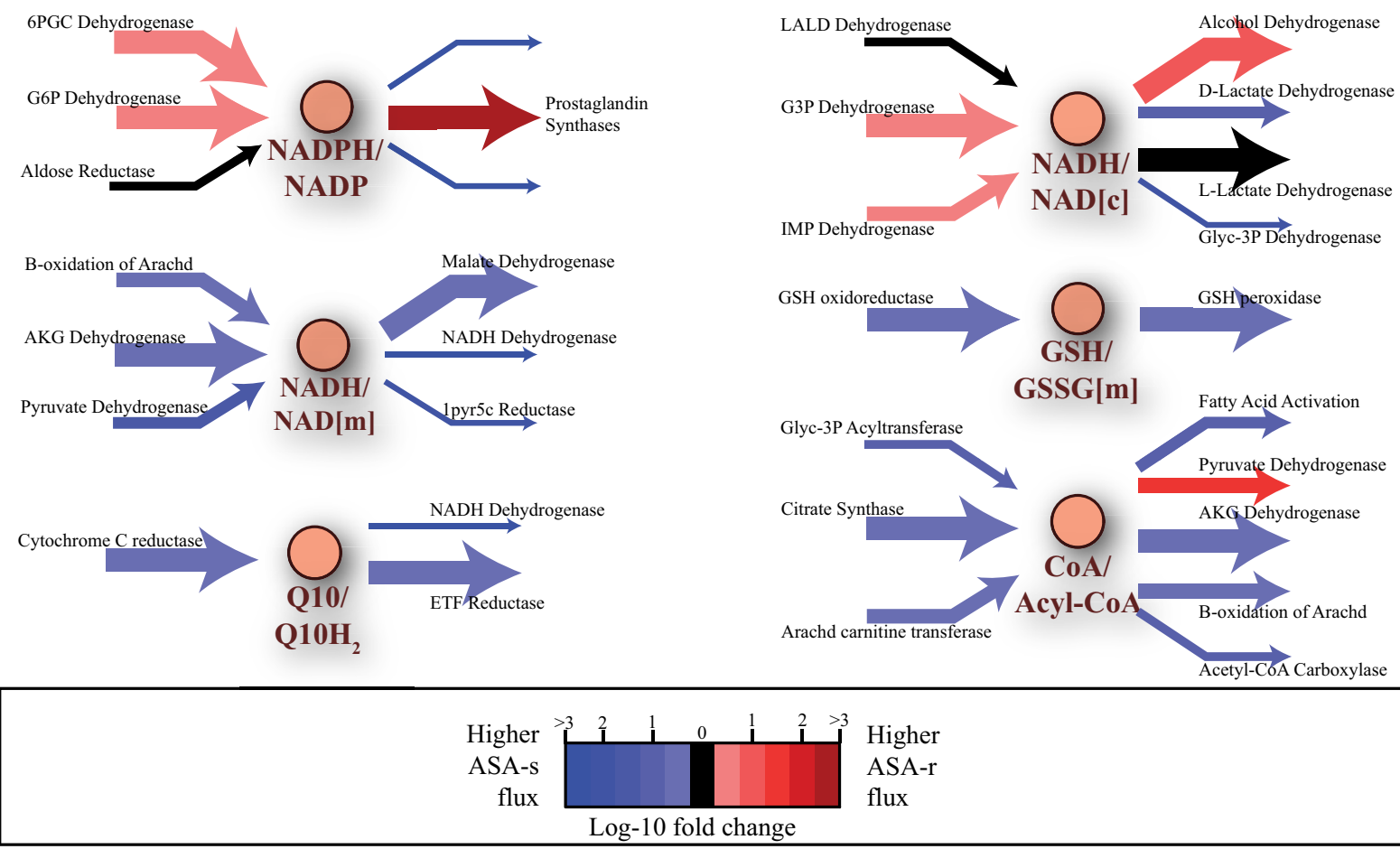

Figure $5 \mid$ Cell-scale view of flux changes in ASA resistant platelet metabolism. (A) Significantly altered fluxes in ASA-resistant versus ASA-sensitive platelet models under a hydrogen peroxide oxidative load after randomized sampling of the solution spaces ${ }^{30}$. Significant (Wilcoxon signed rank-sum test, $p<0.05$ ) flux fold changes are shown as blue reactions and represent reactions with higher flux in the ASA-s phenotype while red reactions have a higher flux in the ASA-r phenotype.(B)Six of the 18 left null space pools for iAT-PLT-636 highlighting key metabolite pools that change in the ASA-r versus ASA-s states. Input/output relations are with respect to the first metabolite listed in the label. The sizes of the reaction arrows represent the flux magnitudes in the ASA-r state. Abbreviations: Txa2 - Thromboxane A2, 6PGC - 6-phospho-D-gluconate, Arachd - arachidonic acid, AKG - 2oxoglutarate, 1pyr5c - 1-pyrroline-5-carboxylate, Q10 - ubiquinone-10, Q10 $\mathrm{H}_{2}$ - ubiquinol-10, ETF - electron transfer flavoprotein, LALD lactaldehyde, G3P - Glyceraldehyde-3-phosphate, Glyc-3P - Glycerol-3-phosphate, IMP - inosine monophosphate, GSH - reduced glutathione, GSSG oxidized glutathione. 
management. This finding is also consistent with the current understanding of cellular responses to oxidative stresses and the need for high concentrations of glutathione in cells, in part for their critical role in counteracting ROS $^{44}$. Furthermore, Mateos-Cáceres et al's results showed no significant change in thioredoxin expression between phenotypes, suggesting intracellular protein oxidants were not a large source of ROS.

An analysis of the left null space pools assisted in the interpretation of the alterations in ASA sensitive versus resistant cases by identifying the flux constraint linking prostaglandin synthesis and central metabolic pathways via the conserved NADP moiety (Figure 5 and Supplement). The results of this study suggest that the metabolic consequences of ASA-resistance on platelet metabolism are elicited by the diversion of metabolites to prostaglandin synthesis and the consequent flux decrease in oxidative metabolism and flux increase in the PPP with corresponding shifts in redox pathways, glycolysis, nucleotide salvage pathways, and lipid metabolism. These pathways have not been previously implicated in ASA-resistance and they present targets for delineating the origins of ASA-resistance. iATPLT-636 represents the current state of knowledge of platelet metabolism. Although the platelet is comparatively simple relative to other human cell types, there is a rich and complex biochemical complement within these cells, and findings in this study and other work suggest that the platelet has an underappreciated role in a wide scope of disease processes. We hope that this functionally integrated, hierarchically structured model of platelet metabolism supports and stimulates further analyses of the platelet and its role in human health and disease.

\section{Methods}

Content curation through comprehensive assessment of the literature (bibliome). 33 proteomic studies on platelets were used as a major source for evidence in enzymatic reactions in the model. Using the 1406 gene transcripts from a generalized human reconstruction ${ }^{14}$ as a reference for genes that participate in human metabolism, the proteomic studies mapped onto 546 unique PDB IDs and their associated 459 Entrez gene IDs in human metabolism. Also, if there was any delineation of the enzyme isoform associated with the PDB ID, it was taken into consideration in the GPR construction. Because platelets are cell fragments of megakaryocytes, it is conceivable that low levels of megakaryocyte gene transcripts and proteins may contribute to noise between proteomic studies. Therefore, mapped gene transcripts that were present in two or more proteomic studies were considered to have enough evidence to be in the model, whereas genes that were present in only one study were considered to have low evidence and were not used in model building. When mapping protein and gene transcripts onto the model, further curation was done to limit the presence of "generalist" enzymes, which promiscuously catalyze reactions on a variety of substrates ${ }^{45}$. Reactions that mapped from generalist enzymes were designated as medium evidence reactions whereas the "specialist" enzyme with proteomic evidence yielded high evidence reactions. After curation efforts were complete on the proteomic studies, 135 reactions had high evidence and 379 reactions had medium evidence. Eventually, evidence indicators were used in the model building algorithm as scoring criteria. In addition to proteomic studies, 289 bibliomic studies were used to justify 142 enzymes in platelet metabolism and 65 bibliomic sources were used to justify 85 extracellular transports. Because bibliomic sources explicitly studied a specific reaction or pathway in platelet metabolism, usually in the context of a disease (or related perturbation) study, the consequent reactions from these sources were all deemed to have high evidence and were included in iAT-PLT636. The logical GPR relationships for each reaction in the model were used to perform the mapping to proteins, SNPs, and individual genes.

Constraints-based modeling approach. The foundation of constraints-based approaches is the stoichiometric representation of the mass- and charge-balanced biochemical transformations of the cell ${ }^{46}$. The core, governing constraints for the model are given by,

$$
S \cdot \vec{v}=0
$$

$$
\vec{v}_{l b}<\vec{v}<\vec{v}_{u b}
$$

where $\mathrm{S}$ is the $m \times n$ stoichiometric matrix for $m$ metabolites and $n$ reactions, $v$ is a $n$ $\times 1$ reaction flux vector and $v_{u b}\left(v_{l b}\right)$ are the upper (lower) reaction bound flux vectors.

Model construction and functional testing. After evidence was assigned to candidate reactions, Model Building Algorithm (MBA) ${ }^{47}$ was used to build a draft model for platelet metabolism. In concordance with the evidence structure for MBA, $\mathrm{C}_{\mathrm{h}}$ is the set of high evidence core reactions, $\mathrm{C}_{\mathrm{m}}$ is the set of medium evidence core reactions, and $R_{p}$ is the set of reactions in the resulting partial model. MBA balances the tradeoff between building a parsimonious model and maximizing the number of reactions with high evidence by selecting the set of reactions meeting the following criteria,

$$
\text { Maximize }\left|\mathrm{R}_{\mathrm{p}} \cap \mathrm{C}_{\mathrm{m}}\right|-\varepsilon\left|\mathrm{R}_{\mathrm{p}} /\left(\mathrm{C}_{\mathrm{h}} \cup \mathrm{C}_{\mathrm{m}}\right)\right| \text { s.t. } \mathrm{C}_{\mathrm{h}} \subset \mathrm{R}_{\mathrm{p}}
$$

$\varepsilon$ is a penalty parameter which balances the tradeoff between building a parsimonious model (high value of $\varepsilon$ ) and maximizing the number of reactions with medium evidence in the model (low value of $\varepsilon$ ). 300 candidate models were generated with MBA to account for alternative reaction pathways that could fulfill the same objective and constraints. In earlier versions of the model construction process, up to 1,000 iterations were used, however we observed that for a model of this size, the reaction content converged by 300 iterations. After the draft model was built, a topological map of the reaction subsystems were drawn using SimPheny software (Genomatica, San Diego, CA) (see Supplement). Reactions with no evidence (used for gap-filling) were verified based on how many high and medium evidence reactions they supported. Therefore, subsystems with low evidence were removed, as they were most likely added as MBA iterated through alternate solutions. The R-group representation of fatty acid metabolism was replaced with the explicit representation of the five most abundant fatty acids in platelets: palmitic (16:0), stearic (18:0), 9-octadecenoic $(18: 1)$, linoleic $(18: 2)$, and arachidonic $(20: 4)$ acids. 300 reactions were added to the model to account for the synthesis, degradation, and interconversion of diacylglycerols, triacylglycerols, inositols, and phospholipids.

Characterization of network dependencies. The different types of functional relationships between reactions shown in Figure 4 were derived by first calculating the flux variability ${ }^{48}$ on the platelet model under open constraints $\left(-1\right.$ to $1000 \mu \mathrm{mol} / 10^{10}$ platelets/hr for all extracellular transporters and -1000 to $1000 \mu \mathrm{mol} / 10^{10}$ platelets/ hr for intracellular reactions). Let $v_{i}$ be a flux through reaction $i$ and let $v_{\min , i}, v_{\text {mid,i }}$ and $v_{\text {max }, i}$ be the minimum, midpoint, and maximum of $v_{i}$ 's flux range. The second step of the method sets the flux through $v_{i}$ at $v_{\text {min }, i}, v_{\text {mid }, i}$ and $v_{\text {max }, i}$ separately and calculates FVA. If the flux through another reaction, $v_{j}$, is uniquely constrained $\left(v_{m i n, j}\right.$ equals $\left.v_{\text {max }, j}\right)$ at the minimum, midpoint, and maximum of $v_{i}$ 's flux, then $v_{i}$ and $v_{j}$ are correlated $^{24}$. Otherwise, if $v_{i}$ constrains the flux $v_{j}$ at any point in its range, then the two reactions are coupled ${ }^{22,49}$ (see comparison in Supplement). Sets of reactions that are perfectly correlated or directionally coupled are in cosets (correlated reaction sets) or coupled sets, respectively. This definition is inherently bi-directional ( $v_{j}$ will constrain $v_{i}$ ). The heatmap in Figure $4 \mathrm{C}$ was created after denoting reaction cosets with a value of 1 and coupled reactions with a value of -1 . Hierarchical clustering is shown employing Jaccard's coefficient and Ward's method. For Figure 4B, reactions in clusters larger than five reactions with Jaccard similarity greater than $75 \%$ were used to represent a condensed biochemical network. The resulting network had 520 of the 1008 reactions in the model.

Construction of ASA-sensitive and ASA-resistant platelet models. The uptake of four carbon sources ([14C]glucose, [14C]acetate, [14C]oleate, and [14C]palmitate) and oxygen, secretion of lactate, and intracellular ATP turnover were used from ${ }^{27}$. Uptakes of $[14 \mathrm{C}]$ citrate $^{25},[14 \mathrm{C}]$ adenine $^{26}$, pyruvate $\mathrm{p}^{15}$, and $[14 \mathrm{C}]$ glutamine ${ }^{28}$ as well as secretion of [14C]hypoxanthine and [14C]xanthine ${ }^{26}$ from other studies in human plasma were used as additional constraints to better characterize energy metabolism in the resting platelet. In order to determine the intracellular flux state of energy metabolism while limiting the total number of participating reactions, $\mathrm{MBA}^{47}$ was once again used to enumerate possible parsimonious flux states for platelet metabolism which enforced the aforementioned exchange constraints and maintained intracellular constraints with respect to measured pathways in platelet energy metabolism. This enumeration allowed for the selection of high likelihood reaction states which could then be used to define minimal media (input reactions), platelet demands (output reactions), and energy pathways (intracellular reactions) that maintained basal platelet energy metabolism. Further constraints on $\mathrm{TXA}_{2}$ secretion and hydrogen peroxide uptake were enforced to define the ASA-sand ASA-r models, with and without an ROS load. To model ASA-r, 95\% of the maximum TXA secretion $\left(1.102 \mu \mathrm{mol} / 10^{10}\right.$ platelets/hr) was set as the lower bound to mimic experimental observations on $\mathrm{TXA}_{2}$ production and ASA-resistivity ${ }^{3}$, whereas the upper bound for $\mathrm{TXA}_{2}$ secretion in ASA-s platelets was set to $1 \%$ of the maximum rate $\left(0.011 \mu \mathrm{mol} / 10^{10}\right.$ platelets/hr $)$ to replicate the irreversible inhibitory effect on $\mathrm{TXA}_{2}{ }^{50}$. The resulting magnitude of the thromboxane secretion constraint lies within physiological limits, as previous studies have measured $0.4^{51}$ to $17.3 \mu \mathrm{mol} /$ $10^{10}$ platelets $/ \mathrm{hr}^{52}$ as the rate of secretion. In order to model the effect of the same load in the context of ASA-resistivity, the upper bound of hydrogen peroxide uptake for both models was set to $95 \%$ of the maximum hydrogen peroxide uptake for the ASAresistant model, which was $13.82 \mu \mathrm{mol} / 10^{10}$ platelets/hr, as the theoretical maximum uptake for the ASA-s model was $6.7 \%$ higher at $14.75 \mu \mathrm{mol} / 10^{10}$ platelets $/ \mathrm{hr}$ hydrogen peroxide. Minimal media and modeling constraints are provided in the supplement. Markov Chain Monte Carlo sampling using the artificial centering hitand-run (ACHR) algorithm was used to uniformly sample the solution space of the constrained platelet models. 5000 sampling points, each representing a feasible flux solution, were simulated over the course of six hours and had a resulting mixed fraction cutoff of $0.51^{30}$. Mixed fractions close to 0.5 indicate uniform sampled points around the median flux value, thus, the sampling of platelet models achieved uniform 
distributions over the solution spaces. The different sampled distributions represent the different phenotypes and significant reaction flux differences were determined using a Wilcoxon signed rank sum test, $p<0.05$. A basis for the left null space of iATPLT-636 was calculated and positive row reduction was performed in order to identify metabolite pools (i.e., sums of metabolites whose sum totals are constant due to the conservation of a particular moiety, e.g. NADP + NADPH). There were 18 of these pools for iAT-PLT-636 (Supplement). Pools with significantly changing reactions determined through the sampling analysis were extracted and were used in the maps for Figure 5B.

1. Antithrombotic Trialists, C. Collaborative meta-analysis of randomised trials of antiplatelet therapy for prevention of death, myocardial infarction, and stroke in high risk patients. BMJ (Clinical research ed.) 324, 71-86 (2002).

2. Wiviott, S. D. \& American Heart Association. Antiplatelet therapy in ischemic heart disease. (Wiley-Blackwell, 2009).

3. Eikelboom, J. W. et al. Aspirin-resistant thromboxane biosynthesis and the risk of myocardial infarction, stroke, or cardiovascular death in patients at high risk for cardiovascular events. Circulation 105, 1650-1655 (2002).

4. Mueller, M. R. et al. Variable platelet response to low-dose ASA and the risk of limb deterioration in patients submitted to peripheral arterial angioplasty. Thromb. Haemost. 78, 1003-1007 (1997).

5. Reed, J. L. Shrinking the metabolic solution space using experimental datasets. PLoS Comput Biol 8, e1002662 (2012).

6. Oberhardt, M. A., Palsson, B. O. \& Papin, J. A. Applications of genome-scale metabolic reconstructions. Mol Syst Biol 5, 320 (2009).

7. Varemo, L., Nookaew, I. \& Nielsen, J. Novel insights into obesity and diabetes through genome-scale metabolic modeling. Front Physiol 4, 92 (2013).

8. Frezza, C. et al. Haem oxygenase is synthetically lethal with the tumour suppressor fumarate hydratase. Nature 477, 225-228 (2011).

9. Chang, R. L., Xie, L., Bourne, P. E. \& Palsson, B. O. Drug off-target effects predicted using structural analysis in the context of a metabolic network model. PLoS Comput Biol 6, e1000938 (2010).

10. Ahn, S. Y. et al. Linkage of organic anion transporter-1 to metabolic pathways through integrated "omics"-driven network and functional analysis. J Biol Chem 286, 31522-31531 (2011)

11. Jamshidi, N., Miller, F. J., Mandel, J., Evans, T. \& Kuo, M. D. Individualized therapy of HHT driven by network analysis of metabolomic profiles. BMC Syst Biol 5, 200 (2011).

12. Ashby, B. Model of prostaglandin-regulated cyclic AMP metabolism in intact platelets: examination of time-dependent effects on adenylate cyclase and phosphodiesterase activities. Mol Pharmacol 36, 866-873 (1989).

13. Bluestein, D., Niu, L., Schoephoerster, R. T. \& Dewanjee, M. K. Steady flow in an aneurysm model: correlation between fluid dynamics and blood platelet deposition. J Biomech Eng 118, 280-286 (1996).

14. Duarte, N. C. et al. Global reconstruction of the human metabolic network based on genomic and bibliomic data. Proc Natl Acad Sci U S A 104, 1777-1782 (2007).

15. Guppy, M., Whisson, M. E., Sabaratnam, R., Withers, P. \& Brand, K. Alternative fuels for platelet storage: a metabolic study. Vox sanguinis 59, 146-152 (1990).

16. Cohen, P., Derksen, A. \& Van den Bosch, H. Pathways of fatty acid metabolism in human platelets. J Clin Invest 49, 128-139 (1970).

17. Reed, J. L., Famili, I., Thiele, I. \& Palsson, B. O. Towards multidimensional genome annotation. Nat Rev Genet 7, 130-141 (2006).

18. Mo, M. L., Jamshidi, N. \& Palsson, B. O. A genome-scale, constraint-based approach to systems biology of human metabolism. Mol Biosyst 3, 598-603 (2007).

19. Amberger, J., Bocchini, C. A., Scott, A. F. \& Hamosh, A. McKusick's Online Mendelian Inheritance in Man (OMIM). Nucleic Acids Res 37, D793-796 (2009).

20. Knox, C. et al. DrugBank 3.0: a comprehensive resource for 'omics' research on drugs. Nucleic Acids Res 39, D1035-1041 (2011).

21. Lewis, N. E., Nagarajan, H. \& Palsson, B. O. Constraining the metabolic genotypephenotype relationship using a phylogeny of in silico methods. Nat Rev Microbiol 10, 291-305 (2012).

22. Burgard, A. P., Nikolaev, E. V., Schilling, C. H. \& Maranas, C. D. Flux Coupling Analysis of Genome-Scale Metabolic Network Reconstructions. Genome. Res. 14, 301-312 (2004).

23. Larhlimi, A. \& Bockmayr, A. A New Approach to Flux Coupling Analysis of Metabolic Networks. Lecture Notes in Computer Science Volume 4216, 205-215 (2006).

24. Jamshidi, N. \& Palsson, B. O. Systems biology of SNPs. Mol Syst Biol 2, 38 (2006).

25. Cartledge, S., Candy, D. J. \& Hawker, R. J. Citrate metabolism by human platelets. Transfusion medicine (Oxford, England) 7, 211-215 (1997).

26. Edenbrandt, C. M. \& Murphy, S. Adenine and guanine nucleotide metabolism during platelet storage at 22 degrees C. Blood 76, 1884-1892 (1990).

27. Guppy, M. et al. Fuel choices by human platelets in human plasma. Eur J Biochem 244, 161-167 (1997).

28. Murphy, S., Munoz, S., Parry-Billings, M. \& Newsholme, E. Amino acid metabolism during platelet storage for transfusion. Br. J. Haematol. 81, 585-590 (1992).

29. Mo, M. L., Palsson, B. O. \& Herrgard, M. J. Connecting extracellular metabolomic measurements to intracellular flux states in yeast. BMC Syst Biol 3, 37 (2009).
30. Schellenberger, J. \& Palsson, B. O. Use of randomized sampling for analysis of metabolic networks. J Biol Chem 284, 5457-5461 (2009).

31. Mateos-Cáceres, P. J. et al. Different expression of proteins in platelets from aspirin-resistant and aspirin-sensitive patients. Thromb. Haemost. 103, 160-170 (2010).

32. Becker, L. B. New concepts in reactive oxygen species and cardiovascular reperfusion physiology. Cardiovasc. Res. 61, 461-470 (2004).

33. Jamshidi, N. \& Palsson, B. O. Using in silico models to simulate dual perturbation experiments: procedure development and interpretation of outcomes. BMC Syst Biol 3, 44 (2009).

34. Famili, I. \& Palsson, B. O. The convex basis of the left null space of the stoichiometric matrix leads to the definition of metabolically meaningful pools. Biophys J 85, 16-26 (2003).

35. Jamshidi, N. \& Palsson, B. O. Top-down analysis of temporal hierarchy in biochemical reaction networks. PLoS Comput Biol 4, e1000177 (2008).

36. Sauro, H. M. \& Ingalls, B. Conservation analysis in biochemical networks: computational issues for software writers. Biophys Chem 109, 1-15 (2004).

37. Papin, J. A., Reed, J. L. \& Palsson, B. O. Hierarchical thinking in network biology: the unbiased modularization of biochemical networks. Trends Biochem Sci 29, 641-647 (2004).

38. David, L., Marashi, S. A., Larhlimi, A., Mieth, B. \& Bockmayr, A. FFCA: a feasibility-based method for flux coupling analysis of metabolic networks. BMC Bioinformatics 12, 236 (2011).

39. Anfossi, G. \& Trovati, M. Role of catecholamines in platelet function: pathophysiological and clinical significance. Eur J Clin Invest 26, 353-370 (1996).

40. Hottz, E. D. et al. Dengue induces platelet activation, mitochondrial dysfunction and cell death through mechanisms that involve DC-SIGN and caspases. J. Thromb. Haemost. 11, 951-962 (2013).

41. Jobe, S. M. et al. Critical role for the mitochondrial permeability transition pore and cyclophilin D in platelet activation and thrombosis. Blood 111, 1257-1265 (2008).

42. Kilkson, H., Holme, S. \& Murphy, S. Platelet metabolism during storage of platelet concentrates at 22 degrees C. Blood 64, 406-414 (1984).

43. Pascale, S. et al. Aspirin-insensitive thromboxane biosynthesis in essential thrombocythemia is explained by accelerated renewal of the drug target. Blood 119, 3595-3603 (2012)

44. Townsend, D. M., Tew, K. D. \& Tapiero, H. The importance of glutathione in human disease. Biomed Pharmacother 57, 145-155 (2003).

45. Nam, H. et al. Network context and selection in the evolution to enzyme specificity. Science 337, 1101-1104 (2012).

46. Orth, J. D., Thiele, I. \& Palsson, B. O. What is flux balance analysis? Nat Biotechnol 28, 245-248 (2010).

47. Jerby, L., Shlomi, T. \& Ruppin, E. Computational reconstruction of tissue-specific metabolic models: application to human liver metabolism. Mol Syst Biol 6, 401 (2010).

48. Mahadevan, R. \& Schilling, C. H. The effects of alternate optimal solutions in constraint-based genome-scale metabolic models. Metab Eng 5, 264-276 (2003).

49. Kim, D. H. \& Motter, A. E. Slave nodes and the controllability of metabolic networks. New J Phys 11, (2009).

50. Vane, J. R. \& Botting, R. M. The mechanism of action of aspirin. Thromb Res $\mathbf{1 1 0}$, 255-258 (2003)

51. Sinha, A. K., Rao, A. K., Willis, J. \& Colman, R. W. Inhibition of thromboxane A2 synthesis in human platelets by coagulation factor Xa. Proc Natl Acad Sci US A 80, 6086-6090 (1983).

52. Butkus, A., Skrinska, V. A. \& Schumacher, O. P. Thromboxane production and platelet aggregation in diabetic subjects with clinical complications. Thromb Res 19, 211-223 (1980)

53. Shannon, P. et al. Cytoscape: a software environment for integrated models of biomolecular interaction networks. Genome Res 13, 2498-2504 (2003).

\section{Acknowledgments}

The authors thank Marc Abrams for his helpful edits and comments of the manuscript. This work was supported by the National Institutes of Health Grant GM068837.

\section{Author contributions}

A.T. drafted the manuscript. A.T. and S.R. reviewed the literature and curated the content. A.T., S.R. and A.B. constructed the models. A.T. and N.J. analyzed the data. N.J., A.T., A.B. and B.O.P. revised the manuscript. N.J. and B.O.P. conceived of the study. All authors read and approved the final content of the manuscript. The authors declare they have no conflict of interest.

\section{Additional information}

Supplementary information accompanies this paper at http://www.nature.com/ scientificreports

Competing financial interests: The authors declare no competing financial interests. 
How to cite this article: Thomas, A., Rahmanian, S., Bordbar, A., Palsson, B.Ø. \& Jamshidi, N. Network reconstruction of platelet metabolism identifies metabolic signature for aspirin resistance. Sci. Rep. 4, 3925; DOI:10.1038/srep03925 (2014). (c) (i) $\Theta$ This work is licensed under a Creative Commons Attribution列 visit http://creativecommons.org/licenses/by-nc-nd/3.0 